\title{
Autorización de la extracción de órganos a fallecidos, una incidencia más del Juzgado de Guardia
}

\author{
Solid organ explant from deceased donor: Consent and police \\ court
}

\begin{abstract}
Resumen
El trasplante de órganos es una de las aportaciones modernas más importantes de la Medicina a la sociedad, y en el curso de este procedimiento interactúan con frecuencia los médicos del equipo de trasplantes (MET) y los médicos forenses (MF), estableciéndose en ocasiones cuestiones o controversias poco comprendidas por el otro colectivo. Así, vemos que algunos MET piensan que aunque el número de negativas judiciales en la extracción de órganos sea pequeño, su trascendencia en el número de receptores es importante, pudiéndose establecer dudas sobre la justificación ética a que una posible sobreprotección de los procedimientos judiciales impida salvar vidas. También se da la circunstancia contraria: algunos MF opinan que el explante de órganos puede imposibilitar la determinación de la causa de la muerte en determinados casos. No son pocos, sin embargo, los facultativos de ambos equipos que opinan que los objetivos de la donación de órganos y la recolección de pruebas forenses no son mutuamente excluyentes. La comprensión de los problemas que se plantean, a una y otra parte, unido a un afán de colaboración adecuado, pueden disminuir el número de negativas. También, la inclusión del MF en los MET podría suponer una significativa mejora en el proceso, tanto médico como judicial, de la extracción y el trasplante de órganos, disminuyendo la negativa judicial de explante de órganos.
\end{abstract}

Palabras clave: Muertes judiciales. Autopsia forense. Trasplante de órganos de cadáver. Especialista en Medicina Legal.

\begin{abstract}
Organ transplantation is one of the most important contributions of modern medicine to society. Surgeons, Physicians and Forensic Doctors often interact in its development, leading rising questions and uncertainties to the rest of the team. For example, many doctors consider that, even the number of judicial refusals on solid organs explants it is reduced, its transcendence on the final number of receptors is important, concluding that an overprotective of the judicial procedures could obstruct save more lives. On the other hand, on the Forensic Doctors view, organ extraction could make impossible determine the cause of death in certain cases. However, all the parts of the team believe that the objectives of organ donation and collection of forensic evidence are not mutually exclusive. Understanding between different professionals, combined with a desire for proper collaboration, can decrease the number of refusals. In addition to that, the inclusion of Forensic Doctors on the solid organ transplant habitual protocols can produce a significant improvement in both medical and judicial process of the removal and transplantation of organs, reducing therefore the judicial negatives to the organ explants.
\end{abstract}

Key words: Judicial deaths. Forensic autopsy. Cadaveric organ transplantation. Forensic medicine expert.

\section{P. Martínez-García P.M. Martínez Pérez-Crespo}

${ }^{1}$ Servicio de Clínica Forense. IML Cádiz ${ }^{2}$ Médico Residente. Hospital Universitario Virgen Macarena. Sevilla

Correspondencia:

Pedro Martínez-García

E-mail: pedro.martinez.garcia@ juntadeandalucia.es
Fecha de recepción: 18.MAY.2015

Fecha de aceptación: 27.MAY.2015 


\section{Introducción}

El trasplante de órganos es una de las aportaciones modernas más importantes de la Medicina a la sociedad, y en el curso de este procedimiento interactúan con frecuencia los médicos del equipo de trasplantes (MET) y los médicos forenses (MF), estableciéndose en ocasiones controversias poco comprendidas por el otro colectivo. Así, vemos que para los MET, aunque el número de negativas judiciales en la extracción de órganos sea pequeño, su trascendencia en el número de receptores es importante, pudiéndose establecer dudas sobre la justificación ética o jurídica al hecho de que una posible sobreprotección de los procedimientos judiciales impida salvar vidas. Frutos et al. ${ }^{1}$ encuentran en una revisión de casos de Andalucía que el 3,6\% del total de extracciones judiciales solicitadas son denegadas (lo que supone un 1,33\% del total de donantes). La distribución en nuestra Comunidad no es uniforme, produciéndose la mayoría de las denegaciones en Málaga y la menor en Sevilla y Almería, lo que puede traducirse por una falta de uniformidad de criterios en el estamento judicial.

También se da la circunstancia contraria. Así, vemos como la Oficina MF del condado de Harris presenta varios casos en los que no ha sido posible determinar la causa de la muerte por la no observancia de los MET de las recomendaciones de los $\mathrm{MF}^{2}$.

No son pocos los facultativos que opinan que los objetivos de la donación de órganos y la recolección de pruebas forenses no son mutuamente excluyentes. Un análisis de los principios y obligaciones éticos de beneficencia, el respeto por la autonomía de la Justicia junto a la comprensión de los problemas que se le plantean, unido a un afán de colaboración adecuado, por ambas partes, pueden disminuir el número de negativas ${ }^{3}$.

\section{Discusión}

Tradicionalmente la clase médica ha considerado que las funciones del MF en el trasplante de órganos son dos: en el caso de donante vivo, certificar las plenas facultades mentales del enfermo, si se cuestiona la validez de su decisión; y en materia de muerte violenta, certificar que la extracción de órganos para la donación no entorpece la investigación para esclarecer la causa de la muerte. En el RD de 1980 se contemplaba que, además del neurólogo o neurocirujano y del Jefe de la Unidad donde estaba el paciente hospitalizado, podría firmar el certificado de defunción el MF si el fallecimiento era judicial, supuesto que no contempla el RD de 1999. Visto así, la tarea parece bastante sencilla e incluso simple; sin embargo, se olvida con frecuencia, en el caso de donante fallecido, que los MF se encuentran vinculados a la LECr.

Antes de entrar en la materia propiamente dicha de este artículo, vamos a ver que en el primer supuesto el médico forense puede ser requerido por el juez para valorar, en caso de enfermedad psíquica, la capacidad de decidir del donante, y con ello la validez del consentimiento. Esta alternativa se trata de una actuación poco frecuente, dado que la valoración tanto clínica como psiquiátrica previa del donante es exhaustiva. El médico deberá informar, a requerimiento del juez, sobre la existencia de alguna enfermedad y como incide esta en la capacidad de conocer o comprender y la libertad de decidir.

En relación a los donantes fallecidos, la intervención del MF se encuentra contemplada, por una parte, en el RD 1723/2012, Art. 9-5: "En los casos de muerte accidental, así como cuando medie una investigación judicial, antes de efectuarse la obtención de órganos deberá recabarse la autorización del juez que corresponda, el cual, previo informe del médico forense, deberá concederla siempre que no se obstaculice el resultado de la instrucción de las diligencias penales"; también, "En los casos de muerte diagnosticada por criterios circulatorios y respiratorios que requieran autorización judicial, para proceder con las maniobras de mantenimiento de viabilidad de los órganos y con las maniobras de preservación, se actuará de conformidad con lo establecido en el anexo I".

$1^{\circ}$ 1b Se han aplicado durante un periodo de tiempo adecuado las medidas de reanimación con resultado infructuoso - reanudar las maniobras de mantenimiento de flujo y comunicarlo al juzgado... tras respuesta favorable o transcurridos 15 minutos iniciar maniobras de preservación, previamente extraer 20 cc de sangre, 20 cc de orina y 20 cc de jugos gástricos.

$2^{\circ}$ 1b No se consideran indicadas las maniobras de reanimación, antes del inicio del procedimiento se comunicará al Juzgado la existencia de un potencial donante, informándole de las circunstancias del caso, y se actuará individualmente de acuerdo con las directrices del caso.

Pero además, como hemos dicho anteriormente, el MF debe ajustar su actuación a la Ley de Enjuiciamiento Criminal ${ }^{5}$, especialmente los artículos 336 y ss, y 757 y ss, que desarrollamos a continuación.

En el Procedimiento Abreviado, que se aplicará al enjuiciamiento de los delitos castigados con pena 
privativa de libertad no superior a nueve años, destacamos el artículo 778, que recoge, en relación a las muertes violentas:

- El informe pericial podrá ser prestado sólo por un perito cuando el Juez lo considere suficiente.

- El Juez podrá acordar, cuando lo considere necesario, que por el médico forense u otro perito se proceda a la obtención de muestras o vestigios cuyo análisis pudiera facilitar la mejor calificación del hecho, acreditándose en las diligencias su remisión al laboratorio correspondiente, que enviará el resultado en el plazo que se le señale.

- El Juez podrá acordar que no se practique la autopsia cuando por el médico forense o quien haga sus veces dictaminen cumplidamente la causa y las circunstancias relevantes de la muerte sin necesidad de aquélla.

- El juez podrá autorizar al médico forense que asista en su lugar al levantamiento del cadáver, adjuntándose en este caso a las actuaciones un informe que incorporará una descripción detallada de su estado, identidad y circunstancias, especialmente todas aquellas que tuviesen relación con el hecho punible.

Vemos, pues, que el MF representa al juez en la diligencia de levantamiento de cadáver, y no podemos olvidar que ésta es una parte primordial, por lo irrepetible, de la autopsia, por cuanto sienta las bases para establecer la naturaleza de la muerte, estimar la etiología medico-legal y orientar la data del fallecimiento. Para Palomo y Ramos ${ }^{6}$, las funciones a desempeñar por el MF en el levantamiento son las siguientes (que exceden en ocasiones la creencia de IOS MET):

- Asegurar el diagnóstico de muerte y la identidad del cadáver.

- Identificar el lugar donde se produjeron las lesiones y el de la muerte. Dado que el hospital es lo que denominamos una escena secundaria, habrá que determinar el lugar donde ocurrieron los hechos, mediante entrevista con los familiares, la policía y la propia historia clínica que puede concretar dónde los servicios de urgencias recogen al paciente.

- Comprobar si el cuerpo fue movido después de la muerte (no procede en estos casos) y explorar las lesiones. Aparte de las lesiones por arma blanca, disparos, contusiones, erosiones, etc. creemos importante hacer especial mención a lesiones figuradas o de difícil explicación que pueda presentar el cadáver. Mención aparte merece el estudio de las manchas de sangre $u$ otros fluidos biológicos que pudieran aparecer en las pertenencias del finado.

- Formular una valoración preliminar del origen y la causa de la muerte por las anotaciones y exploraciones complementarias que obren en la historia clínica. Es imprescindible que el MF que asiste al levantamiento manifieste su opinión "de primera mano" sobre el caso, primero sobre el origen de la muerte (natural, violento o en el supuesto de que haya dudas sobre el mismo indeterminado). También debiera de manifestar su opinión sobre la presumible causa y su impresión sobre la posible intervención de otra persona, accidente o suicidio.

- Formular una valoración preliminar de la data de la muerte.

- Tomar los vestigios del cadáver que pueden perderse o deteriorarse durante el transporte (muestras de sangre, orina, contenido gástrico, hisopos, residuos, etc.). Examinar los vestidos, anotar si presenta algún vestigio orgánico (manchas de sangre, semen, saliva, etc.) u otros como manchas de pintura o vidrio, que deben recogerse o preservarse de forma adecuada para su estudio posterior. Si los vestidos presentan algún daño o alteración, debe valorase su forma de producción, por objetos o muebles colindantes, por armas blancas, cortados por asistencia médica, arrastre del cuerpo, etc.

- Formular una valoración preliminar de la secuencia de los hechos que condujeron a la muerte.

Para ello se pueden realizar fotografías con testigo métrico, esquemas y tomar las muestras necesarias, dado que la protección de manos y cuerpo para evitar la pérdida de vestigios es prácticamente imposible por las maniobras a que va a ser sometido el cuerpo durante las intervenciones para el explante.

Además, el MF tendrá que informar sobre la necesidad de la practica de la autopsia, basándose en criterios médico-legales ${ }^{5}$.

El artículo 343 recoge: "En los sumarios a que se refiere el artículo 340 (muerte violenta o sospechosa de criminalidad), aun cuando por la inspección exterior pueda presumirse la causa de la muerte, se procederá a la autopsia del cadáver por los médicos forenses, o en su caso por los que el juez designe, los cuales, después de describir exactamente dicha operación, informarán sobre el origen del fallecimiento y sus circunstancias. Para practicar la autopsia, se observará lo dispuesto en el artículo 353". El artículo 348 indica que cuando en algún caso, además de la intervención del médico forense, el juez estimase necesaria la cooperación de uno o más facultativos, hará el oportuno nombramiento. 
Lo establecido en el párrafo anterior tendrá también lugar cuando por la gravedad del caso el MF crea necesaria la cooperación de uno o más coprofesores y el juez lo estimare así; alternativa que podría ser utilizada en estas actuaciones, si fuera preciso un segundo perito.

Tras la actuación anterior nos podemos encontrar con tres supuestos según el Código Penal':

- La naturaleza de la muerte es suicida (art. 143): podrá ser necesaria la autopsia si albergamos alguna duda, dado que lo penado en nuestro ordenamiento jurídico es la inducción al suicidio (4 a 8 años) y la cooperación (hasta 10 años).

- La naturaleza de la muerte es accidental (art. 142): puede ser un caso de homicidio imprudente penado con hasta 4 años, en cuyo caso no será necesaria la autopsia si se dictaminan cumplidamente la causa y las circunstancias de la muerte.

- La naturaleza de la muerte es homicida (art. 138 a 141 , penas de 10 hasta 25 años de prisión): será preceptiva la práctica de la autopsia por dos peritos.

La Unión Europea ha emitido una recomendación sobre la autopsia médico-legal que es más estricta que nuestra legislación (Recomendación nº(99) ${ }^{3}$ del Consejo de Ministros de los Estados Miembros, para la Armonización Metodológica de las Autopsias Médico-Legales) ${ }^{8}$ :

- En los casos en que la muerte puede ser debida a causas no naturales, la autoridad competente, acompañada de uno o más peritos médicolegales, deberá investigar adecuadamente el lugar de los hechos, examinar el cuerpo y decidir si debe realizarse la autopsia.

- Se debe practicar la autopsia en todas las muertes no naturales, obvias o sospechosas, también cuando exista demora entre el hecho causal y la muerte, particularmente en los siguientes casos:

- Homicidio o sospecha de homicidio.

- Muerte súbita inesperada, incluyendo el síndrome de muerte súbita del lactante.

- Violación de derechos humanos, como es la sospecha de tortura o cualquier otra forma de maltrato.

- Suicidio o sospecha de suicidio.

- Sospecha de malpraxis médica.

- Accidentes de circulación, laborales o domésticos.

- Enfermedad profesional y ensayos.

- Desastres tecnológicos y naturales.
- Muertes durante detenciones o muertes asociadas con actividades policiales o militares.

- Cuerpos no identificados o restos óseos.

- Los peritos médico-legales deberán realizar sus funciones con total independencia e imparcialidad. No deben estar sometidos a ninguna forma de presión y deben ser objetivos en el ejercicio de sus funciones, especialmente en la presentación de sus resultados y conclusiones.

EI MF se encuentra totalmente vinculado por nuestra legislación (LECr), pero además la recomendación de la Comisión Europea está reconocida por el Ministerio de Justicia y puede hacer albergar la duda sobre el grado de subordinación que el MF tiene con respecto a ella, por lo que se debería dictar alguna recomendación que permitiera conjugar adecuadamente la investigación de la muerte judicial con los trasplantes de órganos.

En el supuesto en que no sea necesaria la práctica de la autopsia, en nuestra opinión, se va a simplificar el problema. El MF emitirá su informe sobre la causa, naturaleza de la muerte y su forma médico-Legal, pronunciándose en el sentido de que la práctica del explante no perjudica la investigación médico-legal, finalizando el escrito con la frase habitual: "no se considera necesaria la práctica de la autopsia, salvo mejor criterio de su señoría dado que dictamina cumplidamente la causa de la muerte".

En el caso de que sea necesaria la autopsia, se tiene que pronunciar en el sentido de qué órgano u órganos son subsidiarios de explantar y cuáles no. Di Maio (Jefe de la Oficina Forense de San Antonio, Texas, y referente mundial en el terreno de la Medicina Legal) y Dana ${ }^{9}$ dicen que la autopsia se hace o no se hace, aunque admiten que "en algún caso de suicidio en los que la bala no ha salido del cuerpo se proceda solo a realizar una autopsia limitada a la extracción de la bala". Además, recomiendan que toda autopsia completa comprenda como mínimo el examen del cerebro; la laringe y el hioides; las vísceras torácicas y abdominales; así como recogida de sangre, bilis, contenido gástrico, orina y vítreo (en casos especiales puede ser necesaria más extensa, como en la tromboembolia pulmonar). Pero la autopsia completa, ¿es totalmente necesaria el día de hoy o los avances en las técnicas de imagen pueden auxiliar a la misma, circunscribiéndola para limitar la pérdida de órganos sin menoscabo de la investigación?

Los beneficios de la autopsia son numerosos y reconocidos por la inmensa mayoría de la comunidad científica. Puede afirmarse que la autopsia es el estudio más completo del enfermo/enfermedad y garantiza la calidad del trabajo médico y la aplicación 
de la justicia ${ }^{10}$. La autopsia es fuente de información genética, alerta sobre riesgos de contagio y otros de carácter personal. Pero la práctica de autopsia alternativa 0 análisis póstumo ${ }^{11}$ va tomando cuerpo poco a poco en la Medicina Clínica. Unas veces por creencias religiosas, otras por las deformaciones estéticas que produce en el cadáver, y por la aparición cada vez más frecuente de enfermedades graves que pueden transmitirse durante su realización (hepatitis $B, C$ y D, sida, etc.). Algunas de esta técnicas, como la autopsia por punciones múltiples, la ecopsia, necro-TAC o tomografía computarizada post mórtem (TAC-PM), necro-RM o resonancia magnética post mórtem (RM-PM) y necroendoscopia o autopsia endoscópica, lamentablemente no están disponibles en los IML de Andalucía, pero sí en los centros hospitalarios en los que se realiza el trasplante. Vemos, pues, que el aumento de la cooperación entre los MF y los MET podría disminuir significativamente la negativa a obtener órganos para trasplantes ${ }^{12}$. Además, afortunadamente nuestra legislación permitiría esta práctica a través del RD 386/96 13 art. 8, ap. 3: "Excepcionalmente, el Director del Instituto podrá autorizar que determinadas prácticas necrópsicas se puedan realizar en lugar distinto de los locales propios del Instituto y de los habilitados con carácter general en el territorio de actuación del Instituto, sin perjuicio de lo establecido en el artículo 353 de la Ley de Enjuiciamiento Criminal"13.

Como se deduce de la exposición anterior, la labor del MF no es cuestión breve y requiere un tiempo, por lo podemos cuestionarnos si el MF dispone de tiempo suficiente para culminar su investigación en los casos de trasplantes de órganos.

En las donaciones por muerte cerebral, el diagnóstico de la muerte se realiza por el cese irreversible de la función neuronal, por un coma arreactivo de etiología conocida y carácter irreversible, en el que debe haber evidencia clínica o por neuroimagen de lesión destructiva en el SNC incompatible con la vida, diagnosticado tras un adecuado periodo de observación. No vamos a describir aquí la exploración a realizar, dado que sobrepasa los límites de este artículo, pero sí destacamos que el periodo de observación (por lo regular de seis horas) puede facilitar la labor del MF.

Ahora bien, ¿qué sucede con la muerte en asistolia? Entre los años 2000 y 2010, debido a iniciativas legislativas y a una mayor concienciación ciudadana, desciende el número de fallecimientos en accidentes de circulación de 14 a 7 diarios. Esta cifra, afortunadamente, sigue bajando a 5 al día en 2012, lo que implica, como efecto colateral, un estancamiento en las donaciones. La situación anterior nos lleva a utilizar nuevas vías, o desarrollar las contempladas en el Decreto de 1999, en beneficio de los pacientes que precisan un trasplante. Estos caminos nos suponen nuevos retos.

El "tiempo de isquemia caliente" (tiempo que transcurre desde la parada cardiaca hasta el establecimiento de las medidas de preservación de los órganos) que sucede a la parada cardiorrespiratoria tiene efectos deletéreos en la viabilidad de los órganos a trasplantar, siendo esta la principal dificultad de este tipo de trasplantes. Este tiempo puede oscilar entre 30 minutos (hígado) y 60 minutos (pulmón, riñón o páncreas).

El diagnóstico de muerte por criterios circulatorios y respiratorios se basará en la constatación de forma inequívoca de ausencia de circulación y de ausencia de respiración espontánea, ambas durante un periodo no inferior a 5 minutos. Y la ausencia de circulación se demostrará mediante la presencia de al menos uno de los siguientes hallazgos:

- Asistolia en un trazado electrocardiográfico continuo.

- Ausencia de flujo sanguíneo en la monitorización invasiva de la presión arterial.

- Ausencia de flujo aórtico en un ecocardiograma. Los protocolos de actuación, derivados de los criterios diagnósticos anteriores, hace que nos podamos encontrar con los siguientes tipos de donantes en asistolia (clasificación de Maastricht):

- Tipo I: fallecido antes de llegar al hospital con tiempo de asistolia conocido. Incluye víctimas de una muerte súbita, traumática o no, acontecida fuera del hospital, que por razones obvias no son resucitadas

- Tipo II: fallecido en el hospital tras maniobras de resucitación infructuosas. (II a parada e inicio de reanimación extrahospitalaria y II b parada ya en el hospital).

- Tipo III: fallecido tras retirada de ventilación mecánica en situaciones de gran daño neurológico irreversible.

- Tipo IV: fallecido en muerte cerebral en el que la asistolia se produce antes de proceder a la extracción.

Estos cuatro tipos han sido encuadrados dentro de dos grandes subgrupos, los denominados "donantes incontrolados", que incluyen el tipo I y II, y los "donantes controlados", que se refiere a los tipos III y IV.

No es preciso señalar las limitaciones que suponen | y |I, no solo temporales, sino simplemente para descartar los criterios de exclusión (causa de muerte 
desconocida, enfermedad infecciosa o tumoral previa, etc.). Pero, además, los protocolos y la logística de los distintos centros en relación a los donantes en asistolia no controlada no es unánime: unos descartan automáticamente las muertes violentas y alguno entra en contradicción. Así, vemos que el Hospital San Carlos y el 12 de Octubre de Madrid, junto con el de San Juan, no excluyen las muertes violentas, mientras que el Clínico de Barcelona, el Virgen de las Nieves y el de La Coruña sí las descartan (aunque algunos entrando en contradicciones) ${ }^{14}$.

Vistos los anteriores escenarios, los MF que ejercen su labor en latitudes tan distintas como Navarra, Murcia o Cataluña ${ }^{15}$ consideran conveniente que los programas de donación de fallecidos por criterios circulatorios y respiratorios que se pongan en marcha en un ámbito territorial, eventualmente contemplen la actuación de un protocolo de comunicación con la autoridad judicial y los IML. Así mismo se debe consensuar la toma de decisiones en situaciones de posible donación en asistolia en los casos de muerte natural no certificada.

En el grado III las posibilidades son mayores, pues se conocen la historia y el proceso inicial del enfermo. En nuestra opinión, habría que estudiar la posibilidad de poder solicitar la autorización judicial una vez que, limitado el esfuerzo terapéutico, se esté en la certeza de hallarse ante un posible donante. El MF podría intervenir recabando toda la información con carácter previo a la muerte, e informar sobre la autorización condicionada al momento de la misma. La limitación temporal también es importante, pero en opinión del perito podría recibirse en el juzgado la comunicación de la existencia de un donante posible (paciente con un daño cerebral grave o paciente con un fallo circulatorio y sin contraindicaciones médicas aparentes para la donación de órganos) o de un donante potencial tipo B (persona en la que se espera el cese de la función circulatoria y respiratoria en un periodo de tiempo que permite la extracción de órganos para trasplante), y solicitar autorización con carácter previo para el caso de que ocurra el óbito, lo que permitiría la actuación previa del MF. Así, la cooperación y la colaboración directa entre los médicos forenses y las organizaciones de trasplantes podría suponer una significativa mejora en el proceso, tanto médico como judicial, de la extracción y trasplante de órganos ${ }^{16}$.

Arslan et al. ${ }^{17}$, tras su estudio de las limitaciones de los casos judiciales en los trasplantes, proponen integrar un experto en medicina forense (FME) en los equipos de trasplantes que siga el proceso de verificación, el muestreo de documento, recogida de muestras, asistencia al explante, fotografía macroscópica e incluso la toma de biopsia. Esto supondría una ventaja para disminuir la pérdida de evidencias y, con toda certeza, responder a la pregunta "icuál fue y cuál no fue la causa de la muerte?", hecho de suma importancia en la investigación judicial. Así se excluiría cualquier situación de la causa de la muerte que pudiera cambiar las características del proceso judicial. Por ejemplo, la ausencia de estudios cardiovasculares en la autopsia de un caso de accidente de tráfico podría cambiar la forma de la muerte y evitar que algunos hallazgos sean "mal utilizados por alguna parte mal intencionada".

Evidentemente, aun así es difícil que el MF pueda desempeñar su labor eficazmente si tiene que atender una agresión sexual y un trasplante en lugares distantes, por lo que en principio debería existir una guardia específica de trasplantes.

\section{Conclusiones}

Se deben poner en marcha territorialmente un protocolo de comunicación con la Autoridad Judicial (Junta de Jueces) y los IML, pudiéndose recibir en el juzgado la comunicación de la existencia de un donante elegible o de un donante potencial tipo B y solicitar autorización con carácter previo para el caso de que ocurra el óbito, lo que permitiría la actuación previa del MF.

Debe aumentar la cooperación directa entre los MF y los MET, estableciendo líneas de colaboración que permitan la realización de exploraciones complementarias, que aunque no tengan significación clínica puedan suponer una evidencia médico-legal de interés para el procedimiento judicial. Así mismo, la inclusión del MF en el equipo de MET podría suponer una significativa mejora en el proceso, tanto médico como judicial, de la extracción y trasplante de órganos, disminuyendo la negativa judicial de explante de órganos.

Sería conveniente la elaboración de un protocolo conjunto nacional (Ministerio de Justicia-ONT), con participación de los MF, que conjugara los criterios de necesidad de autopsia y trasplantes, así como la utilización de técnicas alternativas de autopsia.

Los autores declaran no tener ningún conflicto de intereses. 


\section{Bibliografía}

1. Frutos MA, et al. Profile of organ donors not authorized for judicial reasons. Transplant Proc. 2008;40:287980.

2. Wolf DA, Derrick SM. Undetermined cause and manner of death after organ/tissue donation. Am J Forensic Med Pathol. 2010;31(2):113-6.

3. Shafer $T$, et al. Ethical analysis of organ recovery denials by medical examiners, coroners, and justices of the peace. J Transp/ Coord. 1999;9(4):232-49.

4. Real Decreto $1723 / 2012$ de 28 de diciembre. BOE No 313, de 29 de diciembre de 2012. p. 89315-48.

5. https://www.boe.es/buscar/pdf/1882/BOE-A1882-6036-consolidado.pdf

6. Palomo Jl, Ramos V. Papel del médico forense en la inspección ocular y levantamiento del cadáver. Propuesta de documento. (Recomendaciones, guías, normas o protocolos de actuación profesional). Cuadernos de Medicina Forense. 2004;(36):41-57.

7. Código Penal. Texto consolidado en: https://www.boe. es/buscar/pdf/1995/BOE-A-1995-25444-consolidado.pdf

8. https://www.administraciondejusticia.gob.es/paj/ PA_WebApp_SGNTJ_NPAJ/descarga/5.1-recomendacion n99 ARMOÑIZACION METODOLOGICA DE LAS . pdf? idFile = 21d5e36a-2fb0-43b3-996c$92 \overline{7} \mathrm{e} 6 \mathrm{~d} 2 \mathrm{~d} 3 \mathrm{db} 4$

9. Di Maio VJM, Dana SE. Autopsia forense versus examen externo. En: Manual de patología forense. Madrid: Díaz de Santos; 2003. p. 4.
10. Hurtado de Mendoza J. El futuro de la autopsia. The Electronic Journal of Autopsy. 2005; p. 3-10. http:// rea.uninet.edu

11. García Espinosa B. Generalidades de la autopsia. The Electronic Journal of Autopsy. 2008. p. 4-18. http:// rea.uninet.edu

12. Shafer T, et al. Impact of medical examiner/coroner practices on organ recovery in the United States. JAMA. 1994;272(20):1607-13.

13. RD 386/1996. Reglamento de los Institutos de Medicina Legal. Disponible en: https://www.boe.es/boe/ dias/1996/03/09/pdfs/A09633-09636.pdf

14. Organización Nacional de Trasplantes. Donación en asistolia en España: situación actual y recomendaciones. Documento de Consenso Nacional 2012. p. 83. Disponible en: http://www.ont.es/infesp/DocumentosDeConsenso/DONACI\%C3\%93N\%20EN\%20 ASISTOLIA\% 20EN\%20ESPA \% C3\% 91 A. \% 20 SITUACI\%C3\%93N\%2OACTUAL\%20Y\%20RECOMENDACIONES.pdf

15. Teijeira R, Bañón R, Baberia E. Aspectos de interés médico-legal de la nueva legislación de trasplantes en España. Rev Esp Med Legal. 2013;39(3):93-8.

16. Pérez-Pérez RM, Bardalet-Viñals N, Soler-Murall $N$. Diagnóstico de muerte y trasplante de órganos. Implicaciones jurídicas y médico-legales. Med Clin (Barc). 2006;126(18):707-11.

17. Arslan $\mathrm{MN}$, et al. Limitations of cadaveric organ donation on judicial cases and problems confronted in autopsy: Istanbul data in comparative perspective. Forensic Sci Int. 2014;237:131-6. 